\title{
Identification of the dehydratase component of the mycobacterial mycolic acid-synthesizing fatty acid synthase-II complex
}

\author{
Correspondence \\ Gurdyal S. Besra. \\ g.besra@bham.ac.uk
}

Received 13 August 2007

Revised 10 September 2007

Accepted 12 September 2007

\author{
Alistair K. Brown, $\uparrow$ Apoorva Bhatt, $\uparrow$ Albel Singh, Elesh Saparia, \\ Alex F. Evans and Gurdyal S. Besra
}

School of Biosciences, University of Birmingham, Edgbaston, Birmingham B15 2TT, UK

\section{INTRODUCTION}

Tuberculosis affects a third of the world population and causes 1.8 million fatalities annually (Dye, 2006). The spread of TB has been facilitated in recent years due to the susceptibility of HIV-infected individuals to Mycobacterium tuberculosis, the aetiological agent of TB (Paolo \& Nosanchuk, 2004). The problem has also been compounded by the emergence of multi-drug-resistant TB (MDR-TB) (Kaye \& Frieden, 1996) and extensively drugresistant (XDR)-TB strains (Wright et al., 2006). M. tuberculosis and other mycobacteria have a distinct cell wall which has a lipid-rich outer layer that is highly impermeable (Minnikin, 1982). One of the major components of this outer envelope are mycolic acids, long chain $\alpha$-alkyl, $\beta$-hydroxy fatty acids that are essential for bacterial survival (Bhatt et al., 2005; Parish et al., 2007; Portevin et al., 2004; Vilcheze et al., 2000). These are either found esterified to the non-reducing termini of an arabinogalactan-peptidoglycan cell wall core, or are present in the

†These authors contributed equally to this work.

Abbreviations: ACP, acyl carrier protein; CoA, coenzyme A; DTT, dithiothreitol; FAS, fatty acid synthase; FAMEs, fatty acid methyl esters; MAMEs, mycolic acid methyl esters. free glycolipid trehalose dimycolate (TDM) (Brennan \& Nikaido, 1995; Dover et al., 2004).

The biosynthesis of mycolic acids is linked to the unusual presence of two fatty acid synthases in mycobacteria: a multi-functional mammalian-type fatty acid synthase-I (FAS-I) (Smith et al., 2003), and a bacterial-type multienzyme complex fatty acid synthase-II (FAS-II), in which dissociable enzymes interact with an acyl carrier protein (ACP), AcpM, that manoeuvres the growing fatty acyl chain between their active sites (Kremer et al., 2001). M. tuberculosis FAS-I conducts de novo synthesis of intermediate length (principally $\mathrm{C}_{16}$ and $\mathrm{C}_{24}$ ) fatty acids. On the other hand, FAS-II, while incapable of de novo fatty acid synthesis, extends FAS-I generated primers to long chain fatty acids $\left(\mathrm{C}_{48}-\mathrm{C}_{56}\right)$, denoted as meromycolic acids.

In $M$. tuberculosis, the process is initiated by the condensation of acyl-CoA and malonyl-ACP, a reaction catalysed by $m t \mathrm{FabH}$, a $\beta$-ketoacyl-ACP synthase (Brown et al., 2005). The newly formed $\beta$-ketoacyl-ACP is first reduced by a $\beta$-ketoacyl-ACP-reductase (MabA) (Banerjee et al., 1998) to form a $\beta$-hydroxyacyl-ACP intermediate which is then dehydrated by a yet unidentified $\beta$-hydroxyacyl-ACP-dehydratase to form an enoyl-ACP intermediate. 
This is further reduced by an enoyl-ACP-reductase (InhA) to yield an ACP-bound acyl chain that is two carbons longer (Banerjee et al., 1994; Kikuchi \& Kusaka, 1984). Subsequent reductive FAS-II cycles are initiated by two other $\beta$-ketoacyl-ACP synthases, KasA and KasB, (Kremer et al., 2000; Mdluli et al., 1998; Schaeffer et al., 2001) to form a meromycolic acid. The mero-chain is then condensed with a $\mathrm{C}_{26}$ fatty acid (Gande et al., 2004; Portevin et al., 2005; Takayama et al., 2005) in a reaction catalysed by Pks13, to yield an oxo-mycolic acid intermediate which is then reduced to form a mature mycolic acid (Lea-Smith et al., 2007).

Genes encoding FAS-II enzymes are essential for mycobacterial survival (Bhatt et al., 2005; Parish et al., 2007; Vilcheze et al., 2000); conditional depletion or inactivation of these enzymes leads to bacterial cell lysis (Bhatt et al., 2005; Vilcheze et al., 2000). This makes FAS-II enzymes attractive targets for drug development. Indeed, two wellstudied anti-tubercular agents, isoniazid and thiolactomycin, both target FAS-II enzymes (Banerjee et al., 1994; Kremer et al., 2000). While the genes for most FAS-II enzymes had been identified in two separate loci in the 1990s (Banerjee et al., 1994, 1998; Cole et al., 1998), the ORF encoding the $\beta$-hydroxyacyl-ACP-dehydratase has remained elusive (BLAST searches of E. coli dehydratases FabZ and FabA failed to identify a specific mycobacterial homologue). The first putative dehydratase candidates were identified using bioinformatics in a key study which demonstrated that seven $M$. tuberculosis proteins contained a double hot dog fold closely related to that of (R)-enoyl CoA-hydratase from Aeromonas caviae (PDB code 1IQ6) (Castell et al., 2005). Of these, two genes, Rv3538 and $R v 0636$, were proposed to be essential for mycobacterial growth (Sassetti et al., 2003), though only the latter has a homologue in Mycobacterium leprae which also synthesizes mycolic acids. Rv0636 shares $21 \%$ identity and $37 \%$ similarity with 1IQ6 over the whole protein (Brown et al., 2007), suggesting that Rv0636 was the most likely candidate for the FAS-II dehydratase activity. In a recent study we demonstrated that flavonoid-based inhibitors of dehydratases from Escherichia coli and Plasmodium falciparum were also active against Mycobacterium. bovis BCG (Brown et al., 2007). Growth of M. bovis BCG in the presence of these compounds caused cessation of mycolic acid biosynthesis and growth inhibition. Furthermore, expression of multiple copies of plasmid-borne Rv0636 in $M$. bovis BCG increased its resistance to the flavonoid compounds and restored the biosynthesis of mycolic acids, suggesting that the product of $R v 0636$ was the likely target of these dehydratase inhibitors. The compounds also caused inhibition of Mycobacterium smegmatis FAS-II in a whole-cell in vitro assay (Brown et al., 2007). The bioinformatics analysis and drug inhibition studies together suggested that the putative protein encoded by Rv0636 was likely the FAS-II dehydratase. In this study we chose MSMEG1341, the M. smegmatis homologue of Rv0636, to address the question whether Rv0636 and
MSMEG1341 do indeed encode the mycobacterial FAS-II dehydratase. Utilizing CESTET (conditional expression specialized transduction essentiality test), a previously described method for testing gene essentiality and conditionally depleting essential gene products (Bhatt et al., 2005), we have shown that MSMEG1341 is an essential gene in $M$. smegmatis. Furthermore, the role of MSMEG1341 as the FAS-II $\beta$-hydroxyl-acyl-ACP dehydratase was probed by depleting the enzyme in a conditional mutant.

\section{METHODS}

Bacterial strains, phages, plasmids. All plasmids, phages and bacterial strains used in this study are outlined in Table 1. Strains of $E$. coli were cultured in LB broth. $M$. smegmatis strains were grown in Tryptic Soy Broth (TSB; Difco) containing 0.05\% Tween-80 (TSBT). Solid media were made by adding $1.5 \%$ agar to the above-mentioned broths. The concentrations of antibiotics used were $100 \mu \mathrm{g} \mathrm{ml}^{-1}$ for hygromycin and $20 \mu \mathrm{g} \mathrm{ml}{ }^{-1}$ for kanamycin with $M$. smegmatis and $150 \mu \mathrm{g} \mathrm{ml}{ }^{-1}$ for hygromycin and $40 \mu \mathrm{g} \mathrm{ml}^{-1}$ for kanamycin with E. coli.

Construction of merodiploid strains. The E. coli-Mycobacterium inducible shuttle vector pSD26 containing the acetamidase promoter and encoding a 6-histidine C-terminal tag was used for the construction of pSD26-MSMEG1341 and pSD26-Rv0636, using $M$. smegmatis $\mathrm{mc}^{2} 155$ and M. tuberculosis H37Rv DNA, respectively. All DNA manipulations were performed using standard protocols, as described by Sambrook \& Russell (2001). PCR amplification was performed using the $M$. smegmatis primers $5^{\prime}$-GATCGATCGGATCCATGGCTCTGCGTGAGTT-3' and 5'-GATCGATCGATATCGGCGAGTCGTGCGGTGGCGA-3' and $M$. tuberculosis primers $5^{\prime}$ GATCGATCGGATCCATGGCGCTGCGTGAGTT-3' and 5'-GATCGATCGATATCCGCTAACTTCGCCGA-3', which contain BamHI and EcoRV restriction sites, respectively (underlined). The $454 \mathrm{bp}$ PCR product was then digested with BamHI and EcoRV and ligated with similarly digested pSD26, giving rise to pSD26-MSMEG1341 and pSD26-Rv0636. The coding sequence of the recombinant gene was verified by DNA sequencing. The single-copy-integrating constructs pABMSMEG1341 and pABRv0636 were constructed by ligating approximately $3.2 \mathrm{~kb} X b a \mathrm{I}-\mathrm{ClaI}$ fragments from the pSD26MSMEG1341 and pSD26-Rv0636 constructs (containing the $M$. smegmatis MSMEG1341 and the M. tuberculosis Rv0636 genes cloned in-frame, downstream of the inducible $M$. smegmatis acetamidase promoter) into XbaI-ClaI-digested pMV306. The merodiploid strains $\mathrm{mc}^{2} 155:: \mathrm{pABMSMEG} 1341$ and $\mathrm{mc}^{2} 155:: \mathrm{pABR} 0636$ were obtained by electroporating $\mathrm{mc}^{2} 155$ with $\mathrm{pABMSEG} 1341$ and $\mathrm{pABRv0636}$, respectively, and selecting for kanamycin-resistant colonies (electroporation of $M$. smegmatis was performed as described earlier by Snapper et al., 1990).

Construction of deletion mutants. Approximately $1 \mathrm{~kb}$ sequences of the upstream and downstream regions of MSMEG1341 were PCR amplified from $M$. smegmatis $\mathrm{mc}^{2} 155$ genomic DNA using the primer pairs MS1341LL (5'-TTTTTTTTCCATAAATTGGCTCAGTCGGCAGAGCGTTTC-3') and MS1341LR (5'-TTTTTTTTCCATTTCTT GGTCCCAGTGGATCGGGTTGAG-3'), and MS1341RL (5'-TTTTTTTTCCATAGATTGGTTCAACGGCCGGGTCAAGTC-3') and MS1341RR (5'-TTTTTTTTCCATCTTTTGGTCGTCGCTCACCGCATACTC-3'), respectively. The PCR fragments were digested with Van91I (sites were incorporated in the primers) and cloned into Van91I-digested p0004S (gift from T. Hsu and W. R. Jacobs Jr, Albert Einstein College of Medicine, New York). The recombinant plasmids 
Table 1. Plasmids, bacterial strains, and phages used in this study

\begin{tabular}{|c|c|c|}
\hline Plasmid, strain, or phage & Description & Reference/source \\
\hline \multicolumn{3}{|c|}{ 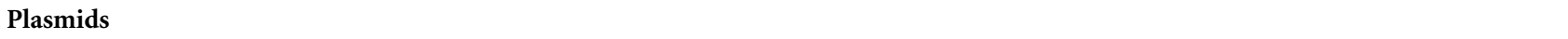 } \\
\hline pMV306 & $\begin{array}{l}\mathrm{Kan}^{\mathrm{R}} \text {, single-copy-integrating vector; inserts into the phage L5 chromosomal } \\
\text { integrating site } \operatorname{att}_{B}{ }^{*} \text { in many mycobacteria }\end{array}$ & Stover et al., 1991 \\
\hline pSD26 & Hyg $^{\mathrm{R}}$, multiple copy, expression vector with the acetamidase promoter & Daugelat et al., 2003 \\
\hline pSD26-MSMEG1341 & M. smegmatis $\mathrm{mc}^{2} 155$ MSMEG1341 cloned into pSD26 & This work \\
\hline pSD26-Rv0636 & M. tuberculosis $\mathrm{H} 37 \mathrm{Rv}$ Rv0636 cloned into pSD26 & This work \\
\hline pABMSMEG1341 & XbaI-ClaI fragment of pSD26-MSMEG1341 subcloned into pMV306 & This work \\
\hline $\mathrm{pABR} v 0636$ & XbaI-ClaI fragment of pSD26-Rv0636 subcloned into pMV306 & This work \\
\hline p0004s & $\begin{array}{l}\text { Vector for cloning allelic-exchange substrates to be used for specialized transduction; } \\
\text { contains } \lambda \text { phage } \cos \text { site and } \operatorname{Hyg}^{\mathrm{R}} \text { marker }(h y g)\end{array}$ & $\begin{array}{l}\text { Gift from Tsungda Hsu } \\
\text { and W. R. Jacobs Jr }\end{array}$ \\
\hline $\mathrm{p} \triangle M S M E G 1341$ & Derivative of p0004s designed for allelic exchange of M. smegmatis MSMEG1341 & This work \\
\hline \multicolumn{3}{|l|}{ Bacterial strains } \\
\hline $\mathrm{mc}^{2} 155$ & Electroporation-proficient ept mutant of $M$. smegmatis strain $\mathrm{mc}^{2} 6$ & Snapper et al., 1990 \\
\hline $\mathrm{mc}^{2} 155:: \mathrm{pABMSMEG1341}$ & $\mathrm{Kan}^{\mathrm{R}} \mathrm{mc}^{2} 155$ derivative containing $\mathrm{pABMSMEG1341}$ integrated into the att $_{B}$ site & This work \\
\hline $\mathrm{mc}^{2} 155:: \mathrm{pABR} v 0636$ & $\mathrm{Kan}^{\mathrm{R}} \mathrm{mc}^{2} 155$ derivative containing $\mathrm{pABRv} 0636$ integrated into the $a t t_{B}$ site & This work \\
\hline$\triangle M S M E G 1341-\mathrm{A}$ & Native copy of MSMEG1341 in $\mathrm{mc}^{2} 155:: \mathrm{pABMSMEG1341}$ is replaced by $h y g$ & This work \\
\hline$\triangle M S M E G 1341-\mathrm{B}$ & Native copy of MSMEG1341 in $\mathrm{mc}^{2} 155:: \mathrm{pABR} v 0636$ is replaced by hyg & This work \\
\hline \multicolumn{3}{|c|}{ 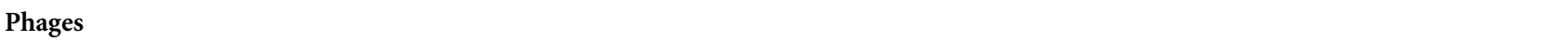 } \\
\hline phAE159 & $\begin{array}{l}\text { Conditionally replicating shuttle phasmid derived from the lytic mycobacteriophage } \\
\text { TM4 }\end{array}$ & Bardarov et al., 2002 \\
\hline $\mathrm{ph} \triangle M S M E G 1341$ & Derivative of phAE159 obtained by cloning p $\triangle M S M E G 1341$ into its unique PacI site & This work \\
\hline
\end{tabular}

${ }^{\star}$ att $_{B}$, Phage L5 chromosomal integration site.

obtained after transforming E. coli TOP-10 cells were sequenced to confirm that there were no errors in the PCR amplified sequences. One plasmid, p $\triangle M S M E G 1341$, was digested with PacI and used for packaging into the temperature sensitive mycobacteriophage phAE159 as described previously (Bardarov et al., 2002) to yield phasmid DNA of the knockout phage ph $\triangle M S M E G 1341$. Generation of high titre phage particles and CESTET were performed as described earlier (Bardarov et al., 2002; Bhatt et al., 2005). PCR verification of allelic exchange was performed using the primer pair MS1341LL and MS1341RR.

Conditional depletion of MSMEG1341. The M. smegmatis strains $\triangle M S M E G 1341-\mathrm{A}$ and $\triangle M S M E G 1341-\mathrm{B}$ were grown in TSBT and $0.2 \%$ acetamide to an $\mathrm{OD}_{600}$ of 0.5 . The cells were washed twice in TSBT to remove traces of acetamide and resuspended to the original volume in TSBT. This cell suspension was used as a $20 \%$ inoculum in TSBT and grown for $12 \mathrm{~h}$ to deplete intracellular MSMEG1341/ Rv0636. The depleted culture was then used to inoculate TSBT with or without $0.2 \%$ acetamide ( $5 \%$ inoculum). This inoculation point of the depleted culture corresponds to the time ' 0 ' for all experiments designed to test cell density, viability and mycolate and lipid profiles.

Determination of the in vivo effects of MSMEG1341 depletion on fatty acid and mycolic acid synthesis. Samples of $\triangle M S M E G 1341$-A grown in the presence or absence of acetamide were taken at time points $0,6,12,24 \mathrm{~h}$ and labelled with $1 \mu \mathrm{Ci} \mathrm{ml} l^{-1}$ $\left(37 \mathrm{kBq} \mathrm{ml}^{-1}\right)\left[1,2{ }^{14} \mathrm{C}\right]$ acetate $\left[57 \mathrm{mCi} \mathrm{mmol}^{-1}\left(2.1 \mathrm{GBq} \mathrm{mmol}^{-1}\right)\right.$, GE Healthcare, Amersham Bioscience], followed by incubation at $37{ }^{\circ} \mathrm{C}$ for $3 \mathrm{~h}$. The ${ }^{14} \mathrm{C}$-labelled cells were harvested by centrifugation at $2000 \mathrm{~g}$ followed by washing with PBS. The cell pellet was subjected to alkaline hydrolysis using $5 \%$ aqueous tetrabutylammonium hydroxide (TBAH) at $100{ }^{\circ} \mathrm{C}$ overnight, followed by the addition of $4 \mathrm{ml} \mathrm{CH}_{2} \mathrm{Cl}_{2}, 500 \mu \mathrm{l} \mathrm{CH}_{3} \mathrm{I}$ and $2 \mathrm{ml}$ water, followed by mixing for $30 \mathrm{~min}$. The upper aqueous phase was discarded following centrifugation and the lower organic phase washed thrice with water and evaporated to dryness. The resulting fatty acid methyl esters (FAMEs) and mycolic acid methyl esters (MAMEs) were dissolved in diethyl ether, insoluble residues were removed by centrifugation and the ether solution evaporated to dryness and redissolved in $200 \mu \mathrm{l}$ $\mathrm{CH}_{2} \mathrm{Cl}_{2}$. Equivalent counts (25000 c.p.m.) of the resulting solution of FAMEs and MAMEs were subjected to thin-layer chromatography (TLC) using silica gel plates ( 5735 silica gel $60 \mathrm{~F}_{254}$; Merck), developed in petroleum ether-acetone $(95: 5)$. Autoradiograms were produced by overnight exposure of Kodak X-Omat AR film to the plates to reveal ${ }^{14} \mathrm{C}$-labelled FAMEs and MAMEs.

\section{RESULTS}

\section{Bioinformatic analysis of Rv0636 and flanking genes}

The putative peptide encoded by $R v 0636$ showed the presence of a hydratase-2 motif (G-D-X-N-P-L-I-V-H-X $5_{5}^{-}$ A) found in the 'lid' region of hot dog fold-containing dehydratases (Castell et al., 2005), and included the catalytic residues [D-X $\mathrm{X}_{4}-\mathrm{H}$ ] (Qin et al., 2000). Structure predictions based on A. caviae dehydratase crystal structure 1IQ6 revealed that Rv0636 has the hydratase-2 motifcontaining 'lid' in a similar orientation as 1IQ6, E. coli FabA and P. falciparum FabZ, consistent with the hot dog fold postulated to be necessary for dehydratase activity (data not shown). A closer analysis of genomic sequences flanking $R v 0636$ indicated that the gene may be cotranscribed with $R v 0635$ and $R v 0637$ : the former ORF 
overlaps with $R v 0636$ while the start codon of $R v 0637$ is only $3 \mathrm{bp}$ downstream of the stop codon of $R v 0636$. Indeed, the operon prediction website of The Institute of Genomic Research, USA, lists the genes as part of a predicted operon (http://www.tigr.org/tigr-scripts/operons/operons.cgi). Interestingly, while neither flanking gene shows any similarity to $R v 0636$, the putative $R v 0635$ encoded peptide is $48 \%$ identical and $65 \%$ similar to that encoded by $R v 0637$. While $R v 0635$, like $R v 0636$, is an essential gene, $R v 0637$ is not essential for growth (Sassetti et al., 2003). These findings suggested that $R v 0635, R v 0636$ and $R v 0637$ may be functionally related, and that $R v 0635$ and $R v 0637$ may encode similar functions. Furthermore, these genes are situated close to the mmaA1-mmaA4 cluster involved in biosynthesis of oxygenated mycolic acids. MSMEG1341 is the M. smegmatis homologue of $R v 0636$ and both $M$. smegmatis and $M$. leprae show a similar arrangement of ORFs to those found in the $R v 0635-R v 0637$ region of $M$. tuberculosis (Fig. 1). We chose the $M$. smegmatis gene MSMEG1341 for further genetic analysis of this gene cluster.

\section{MSMEG1341 is essential in M. smegmatis}

Specialized transduction of $M$. smegmatis $\mathrm{mc}^{2} 155$ with ph $\triangle M S M E G 1341$, a temperature-sensitive phage containing an allelic-exchange substrate designed to replace MSMEG1341 with a hygromycin resistance cassette (hyg), failed to yield any hygromycin-resistant $\left(\mathrm{Hyg}^{\mathrm{R}}\right)$ transductants on TSB agar (TSBA) (data not shown), suggesting that MSMEG1341 was essential for the growth of $M$. smegmatis. CESTET (Bhatt et al., 2005) was used to confirm the essentiality of MSMEG1341. Firstly, a merodiploid strain was generated by integration of pABMSMEG1341 into the M. smegmatis $\mathrm{mc}^{2} 155$ chromosome. The plasmid is a single copy integrative vector that contains MSMEG1341 cloned downstream of the acetamide-induced acetamidase promoter (Mahenthiralingam et al., 1993). The resultant strain $\mathrm{mc}^{2} 155:$ : pABMSMEG1341, when transduced with ph $\triangle M S M E G 1341$, yielded $\mathrm{Hyg}^{\mathrm{R}}$ colonies only on TSBA plates containing acetamide. The replacement of the native chromosomal copy of MSMEG1341 in these transductants was con-

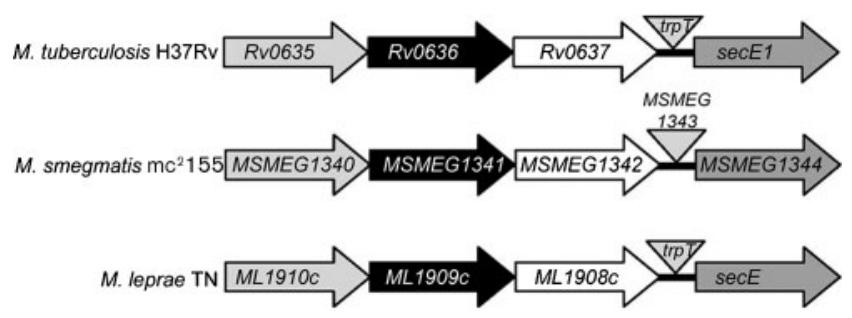

Fig. 1. Maps of the genomic regions of M. tuberculosis Rv0636, M. smegmatis $\mathrm{mc}^{2} 155$ MSMEG1341 and M. leprae TN ML1909. The inverted triangles represent tRNA-encoding genes. (a) $\mathrm{mc}^{2} 155:: \mathrm{pABMSMEG1341}$

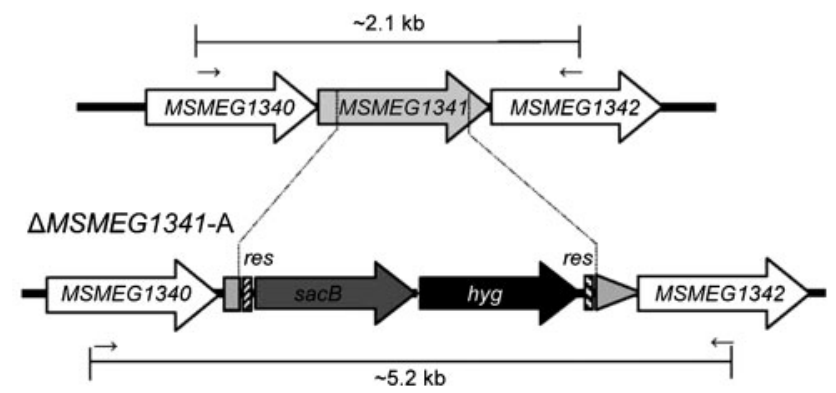

(b)

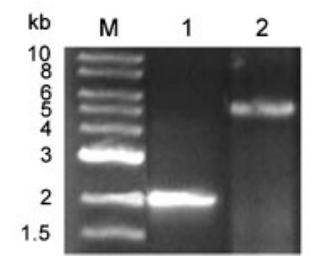

Fig. 2. (a) Map of the MSMEG1341 region in the $M$. smegmatis $\mathrm{mc}^{2} 155:: \mathrm{pABMSMEG1341}$ genome and its corresponding region in the conditional mutant $\triangle M S M E G 1341-\mathrm{A}$. res, $\gamma \delta$ resolvase site; hyg, hygromycin resistance gene from Streptomyces hygroscopicus; sacB, sucrose counterselectable gene from Bacillus subtilis. (b) Agarose gel electrophoresis of PCR products obtained from genomic DNA isolated from $\mathrm{mc}^{2} 155$ (lane 1) or $\triangle M S M E G 1341-A$ (lane 2); M, DNA size marker. The primers used for PCR amplification are indicated by small arrows in the map and the expected sizes of the PCR products are indicated with straight lines.

firmed by PCR (Fig. 2) and Southern blot (data not shown). One transductant was chosen for further analysis and was denoted $\triangle M S M E G 1341-\mathrm{A}$. Subculture of $\triangle M S M E G 1341-\mathrm{A}$ on TSBA with or without acetamide showed that strain could grow only in the presence of acetamide (Fig. 3a), demonstrating that acetamidasepromoter-driven expression of the non-native copy of MSMEG1341 in $\triangle M S 1341$-A was essential for its survival, thus confirming the essentiality of MSMEG1341.

\section{Conditional depletion of MSMEG1341 causes mycobacterial cell lysis}

Previous studies demonstrated that conditional depletion of two FAS-II enzymes, InhA and KasA, in CESTETderived conditional M. smegmatis mutants resulted in cell lysis (Bhatt et al., 2005). If MSMEG1341 was the FAS-II dehydratase, loss of its activity would be expected to result in cell lysis. The growth of $\triangle M S M E G 1341-\mathrm{A}$ in liquid medium with or without acetamide was monitored over $24 \mathrm{~h}$. While the strain grew normally in medium containing acetamide, the culture in the medium without acetamide showed a decrease in $\mathrm{OD}_{600}$ values with time (data not shown), resulting in a clearly lysed culture after $24 \mathrm{~h}$ of incubation (Fig. 3c). Monitoring of viable counts 
(a)

+ Acetamide

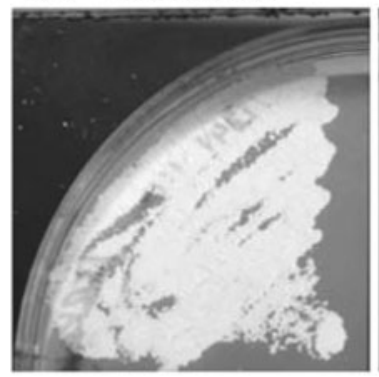

(b)

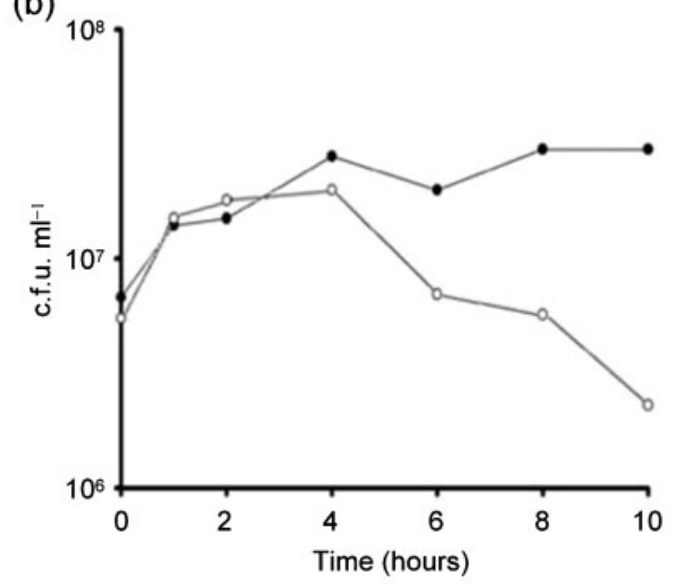

(c)

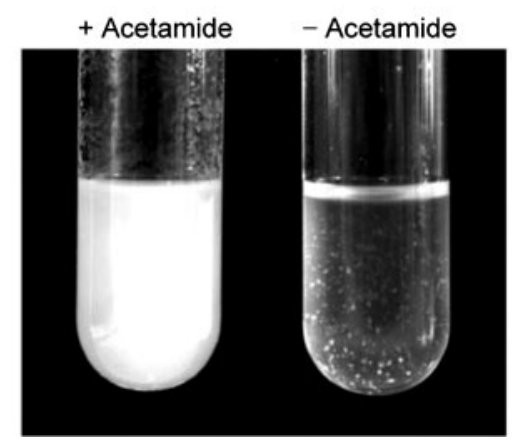

Fig. 3. (a) Growth of $\triangle M S M E G 1341-\mathrm{A}$ on TSB agar with or without acetamide. Growth of the strain is dependent on the acetamide inducible expression of the second recombinant copy of MSMEG1341, thus demonstrating the essentiality of MSMEG1341 for growth of $M$. smegmatis. (b) Plot of viable counts (c.f.u.) of $\triangle M S M E G 1341-\mathrm{A}$ grown in TSBT with $(\bullet)$ or without $(\bigcirc)$ acetamide. (c) Pictures of the cultures of $\triangle M S M E G 1341-\mathrm{A}$ grown for $24 \mathrm{~h}$ in TSBT with or without acetamide.

demonstrated that the culture grown in the absence of acetamide showed a decrease in viability. After $10 \mathrm{~h}$ of growth the culture grown in the absence of acetamide had more than a log fewer colony forming units than that grown in the presence of acetamide (Fig. 3b). This result indicated that, like other FAS-II enzymes, depletion of MSMEG1341 was bactericidal and resulted in cell lysis, making it likely that MSMEG1341 was the FAS-II dehydratase.

\section{Loss of MSMEG1341 results in cessation of mycolic acid biosynthesis}

Loss of FAS-II dehydratase activity would be expected to lead to a loss of mycolic acid biosynthesis. To assess the effects of depletion of MSMEG1341 in the conditional mutant, we pulsed cultures of $\triangle M S 1341-\mathrm{A}$ with $\left[{ }^{14} \mathrm{C}\right]$ acetate at different time intervals after inoculation into TSBT with or without acetamide. Total mycolic acids were extracted from the pulsed cultures as MAMEs and separated by TLC. While the culture grown in the presence of acetamide showed no alterations in the levels of newly synthesized mycolates, a decrease was observed in the culture grown in the absence of acetamide within $6 \mathrm{~h}$ of growth (Fig. 4). The decrease in the latter was not accompanied by a decrease in the level of labelled FAS-Isynthesized fatty acids up to $12 \mathrm{~h}$ (Fig. 4). These results clearly demonstrated the role of MSMEG1341 in mycolic acid biosynthesis. In addition, the above phenotype of MAMEs isolated either from chloroform/methanol lipid extracts or from delipidated cells (i.e. cell wall-bound MAMEs) afforded similar results (data not shown).

\section{Rv0636 is the functional equivalent of MSMEG1341 in M. tuberculosis}

While sequence comparisons and the corresponding organization of flanking genes in M. tuberculosis and $M$. smegmatis indicated that Rv0636 and MSMEG1341 were homologues, it was necessary to confirm that the products of both genes were functionally equivalent. To this end, we introduced Rv0636 into M. smegmatis $\mathrm{mc}^{2} 155$ by single copy integration of the plasmid pABRv0636. Similar to pABMSMEG1341, the expression of $R v 0636$ could be controlled by the acetamidase promoter. The resulting strain $\mathrm{mc}^{2} 155: \mathrm{pABR} v 0636$ was then transduced with ph $\triangle M S M E G 1341$. A Hyg $^{\mathrm{R}}$ transductant obtained on an acetamide-containing plate was analysed by PCR and was found to contain a deletion of MSMEG1341, replacing it

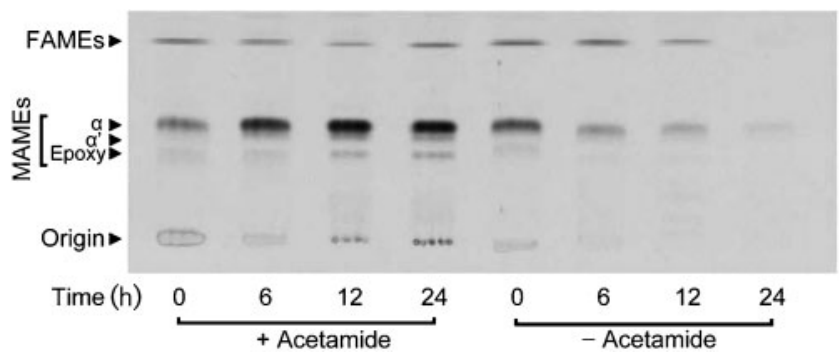

Fig. 4. Autoradiograph (Kodak X-Omat AR film) exposed to TLC plate used for separating ${ }^{14} \mathrm{C}$-labelled methyl esters of mycolic acids (MAMEs) extracted from cultures of $\triangle M S M E G 1341-\mathrm{A}$ grown in TSBT with or without acetamide (extraction and TLC protocols are described in Methods). $\alpha, \alpha^{\prime}$ and epoxy correspond to $\alpha$-mycolates, $\alpha^{\prime}$-mycolates and epoxy mycolates respectively. FAMEs, fatty acid methyl esters. 
with hyg (data not shown). Furthermore, the strain (denoted $\Delta M S 1341-\mathrm{B}$ ), was unable to grow on a plate lacking acetamide on subsequent subculture (Fig. 5a), and growth in liquid medium devoid of acetamide led to cell lysis (Fig. 5b). These data demonstrated that $R v 0636$ could functionally complement the putative M. smegmatis FAS-II dehydratase and therefore by synergy be denoted as the $M$. tuberculosis FAS-II dehydratase.

\section{DISCUSSION}

When the genes encoding KasA/B, MabA and InhA, the core enzymes of the FAS-II reductive cycle, were first identified in two different regions of the M. tuberculosis chromosome (Banerjee et al., 1998; Cole et al., 1998), no ORF encoding a dehydratase was detected in either locus. Following the identification of an R-specific hydratase hot dog fold in M. tuberculosis Rv0636 (Castell et al., 2005), we demonstrated that overexpression of Rv0636 in mycobacteria conferred resistance to known dehydratase inhibitors (Brown et al., 2007). Thus, the data available from bioinformatics-based studies and drug inhibition experiments collectively suggested that Rv0636 was the FAS-II $\beta$ hydroxyacyl-ACP-dehydratase required for mycolic acid biosynthesis. Subsequently, we selected the M. smegmatis gene MSMEG1341, the homologue of M. tuberculosis Rv0636, for generating a mutant strain. Using the conditional mutant $\triangle M S M E G 1341$ we demonstrated that M. smegmatis MSMEG1341 was an essential gene and that, like other FAS-II enzymes (Bhatt et al., 2005; Vilcheze et al.,

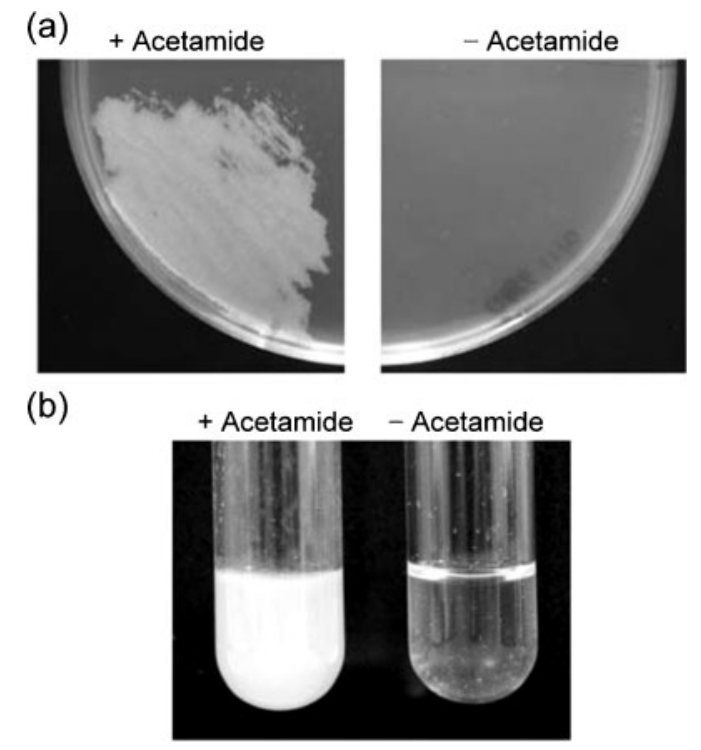

Fig. 5. (a) Growth of MSMEG1341 knockout generated in a strain expressing an acetamide-inducible copy of $M$. tuberculosis Rv0636 (AMSMEG1341-B) on TSB agar plates with or without acetamide. (b) Cultures of the same strain in TSBT with or without acetamide after $24 \mathrm{~h}$ of incubation.
2000), depletion of MSMEG1341 led to loss of viability and caused cell lysis. Loss of MSMEG1341 was also shown to correlate with a cessation of mycolic acid biosynthesis. Together, the molecular modelling data, essentiality of MSMEG1341 and the loss of mycolic acid biosynthesis and subsequent lysis following depletion in a conditional MSMEG1341 mutant established that MSMEG1341 encoded the FAS-II dehydratase component in $M$. smegmatis. Furthermore, null MSMEG1341 mutants could also be generated in an $M$. smegmatis strain containing an inducible copy of M. tuberculosis Rv0636, confirming that Rv0636 was the functional homologue of MSMEG1341 and was thus the M. tuberculosis FAS-II dehydratase.

Due to the emergence of MDR-TB (Kaye \& Frieden, 1996), and more recently XDR-TB (Wright et al., 2006), it has become imperative to look for new drug targets. Due to the essential nature of mycolic acids in mycobacterial virulence and survival, enzymes involved in the biosynthesis of these unique fatty acids have always been attractive targets for drug development (Schroeder et al., 2002). Isoniazid and ethionamide target InhA and derivatives of thiolactomycin have been tested against KasA (Banerjee et al., 1994; Kremer et al., 2000). With the identification of the dehydratase, an additional component of FAS-II can now be targeted for development of drugs that inhibit mycolic acid biosynthesis. Indeed, as mentioned above, certain flavonoid-derived compounds have already been tested and found to inhibit FAS-II activity (Brown et al., 2007). Our identification of M. tuberculosis Rv0636 and M. smegmatis MSMEG1341 as the FAS-II dehydratase completes the identification of all the genes encoding the core FAS-II enzymes and opens up the possibility of testing compounds known to target dehydratases in other bacteria. The development of an in vitro assay for Rv0636 activity would greatly facilitate these studies.

\section{ACKNOWLEDGEMENTS}

The authors would like to thank T. Hsu, J. Kriakov and W. R. Jacobs Jr, Albert Einstein College of Medicine, New York for the generous gift of p0004S and phAE159. G. S. B. acknowledges support in the form of a Royal Society Wolfson Research Merit Award and a Personal Research Chair from Mr James Bardrick, as a former Lister Institute-Jenner Research Fellow, the Medical Research Council (UK), and the Wellcome Trust. A.B. acknowledges support from the Medical Research Council (UK) in the form of a Career Development Award.

\section{REFERENCES}

Banerjee, A., Dubnau, E., Quemard, A., Balasubramanian, V., Um, K. S., Wilson, T., Collins, D., de Lisle, G. \& Jacobs, W. R., Jr (1994). inhA, a gene encoding a target for isoniazid and ethionamide in Mycobacterium tuberculosis. Science 263, 227-230.

Banerjee, A., Sugantino, M., Sacchettini, J. C. \& Jacobs, W. R., Jr (1998). The mabA gene from the inhA operon of Mycobacterium tuberculosis encodes a 3-ketoacyl reductase that fails to confer isoniazid resistance. Microbiology 144, 2697-2704. 
Bardarov, S., Bardarov, S., Pavelka, M. S., Sambandamurthy, V., Larsen, M., Tufariello, J., Chan, J., Hatfull, G. \& Jacobs, W. R. (2002). Specialized transduction: an efficient method for generating marked and unmarked targeted gene disruptions in Mycobacterium tuberculosis, M. bovis BCG and M. smegmatis. Microbiology 148, 3007-3017.

Bhatt, A., Kremer, L., Dai, A. Z., Sacchettini, J. C. \& Jacobs, W. R., Jr (2005). Conditional depletion of KasA, a key enzyme of mycolic acid biosynthesis, leads to mycobacterial cell lysis. J Bacteriol 187, 7596-7606.

Brennan, P. J. \& Nikaido, H. (1995). The envelope of mycobacteria. Annu Rev Biochem 64, 29-63.

Brown, A. K., Sridharan, S., Kremer, L., Lindenberg, S., Dover, L. G., Sacchettini, J. C. \& Besra, G. S. (2005). Probing the mechanism of the Mycobacterium tuberculosis $\beta$-ketoacyl-acyl carrier protein synthase III $m t \mathrm{FabH}$ : factors influencing catalysis and substrate specificity. J Biol Chem 280, 32539-32547.

Brown, A. K., Papaemmanouil, A., Bhowruth, V., Bhatt, A., Dover, L. G. \& Besra, G. S. (2007). Flavonoid inhibition as novel antimycobacterial agents targeting Rv0636, a putative dehydratase enzyme involved in Mycobacterium tuberculosis fatty acid synthaseII. Microbiology 153, 3314-3322.

Castell, A., Johansson, P., Unge, T., Jones, T. A. \& Backbro, K. (2005). Rv0216, a conserved hypothetical protein from Mycobacterium tuberculosis that is essential for bacterial survival during infection, has a double hotdog fold. Protein Sci 14, 1850-1862.

Cole, S. T., Brosch, R., Parkhill, J., Garnier, T., Churcher, C., Harris, D., Gordon, S. V., Eiglmeier, K., Gas, S. \& other authors (1998). Deciphering the biology of Mycobacterium tuberculosis from the complete genome sequence. Nature 393, 537-544.

Daugelat, S., Kowall, J., Mattow, J., Bumann, D., Winter, R., Hurwitz, R. \& Kaufmann, S. H. (2003). The RD1 proteins of Mycobacterium tuberculosis: expression in Mycobacterium smegmatis and biochemical characterization. Microbes Infect 5, 1082-1095.

Dover, L. G., Cerdeno-Tarraga, A. M., Pallen, M. J., Parkhill, J. \& Besra, G. S. (2004). Comparative cell wall core biosynthesis in the mycolated pathogens, Mycobacterium tuberculosis and Corynebacterium diphtheriae. FEMS Microbiol Rev 28, 225-250.

Dye, C. (2006). Global epidemiology of tuberculosis. Lancet 367, 938-940.

Gande, R., Gibson, K. J., Brown, A. K., Krumbach, K., Dover, L. G., Sahm, H., Shioyama, S., Oikawa, T., Besra, G. S. \& Eggeling, L. (2004). Acyl-CoA carboxylases (accD2 and accD3), together with a unique polyketide synthase $(C g-p k s)$, are key to mycolic acid biosynthesis in Corynebacterianeae such as Corynebacterium glutamicum and Mycobacterium tuberculosis. J Biol Chem 279, 44847-44857.

Kaye, K. \& Frieden, T. R. (1996). Tuberculosis control: the relevance of classic principles in an era of acquired immunodeficiency syndrome and multidrug resistance. Epidemiol Rev 18, 52-63.

Kikuchi, S. \& Kusaka, T. (1984). Purification of NADPH-dependent enoyl-CoA reductase involved in the malonyl-CoA dependent fatty acid elongation system of Mycobacterium smegmatis. J Biochem (Tokyo) 96, 841-848.

Kremer, L., Douglas, J. D., Baulard, A. R., Morehouse, C., Guy, M. R., Alland, D., Dover, L. G., Lakey, J. H., Jacobs, W. R., Jr \& other authors (2000). Thiolactomycin and related analogues as novel antimycobacterial agents targeting KasA and KasB condensing enzymes in Mycobacterium tuberculosis. J Biol Chem 275, 16857-16864.

Kremer, L., Nampoothiri, K. M., Lesjean, S., Dover, L. G., Graham, S., Betts, J., Brennan, P. J., Minnikin, D. E., Locht, C. \& Besra, G. S. (2001). Biochemical characterization of acyl carrier protein (AcpM) and malonyl-CoA: AcpM transacylase (mtFabD), two major components of Mycobacterium tuberculosis fatty acid synthase II. J Biol Chem 276, 27967-27974.

Lea-Smith, D. J., Pyke, J. S., Tull, D., McConville, M. J., Coppel, R. L. \& Crellin, P. K. (2007). The reductase that catalyzes mycolic motif synthesis is required for efficient attachment of mycolic acids to arabinogalactan. J Biol Chem 282, 11000-11008.

Mahenthiralingam, E., Draper, P., Davis, E. O. \& Colston, M. J. (1993). Cloning and sequencing of the gene which encodes the highly inducible acetamidase of Mycobacterium smegmatis. J Gen Microbiol 139, 575-583.

Mdluli, K., Slayden, R. A., Zhu, Y., Ramaswamy, S., Pan, X., Mead, D., Crane, D. D., Musser, J. M. \& Barry, C. E., III (1998). Inhibition of a Mycobacterium tuberculosis $\beta$-ketoacyl ACP synthase by isoniazid. Science 280, 1607-1610.

Minnikin, D. E. (1982). Lipids: complex lipids, their chemistry, biosynthesis and roles. In The Biology of the Mycobacteria: Physiology, Identification and Classification. Edited by C. Ratledge \& J. Stanford. London, UK: Academic Press.

Paolo, W. F., Jr \& Nosanchuk, J. D. (2004). Tuberculosis in New York city: recent lessons and a look ahead. Lancet Infect Dis 4, 287-293.

Parish, T., Roberts, G., Laval, F., Schaeffer, M., Daffe, M. \& Duncan, K. (2007). Functional complementation of the essential gene fabG1 of Mycobacterium tuberculosis by Mycobacterium smegmatis fabG but not Escherichia coli fabG. J Bacteriol 189, 3721-3728.

Portevin, D., De Sousa-D’Auria, C., Houssin, C., Grimaldi, C., Chami, M., Daffe, M. \& Guilhot, C. (2004). A polyketide synthase catalyzes the last condensation step of mycolic acid biosynthesis in mycobacteria and related organisms. Proc Natl Acad Sci U S A 101, 314-319.

Portevin, D., de Sousa-D’Auria, C., Montrozier, H., Houssin, C., Stella, A., Laneelle, M. A., Bardou, F., Guilhot, C. \& Daffe, M. (2005). The acyl-AMP ligase FadD32 and AccD4-containing acyl-CoA carboxylase are required for the synthesis of mycolic acids and essential for mycobacterial growth: identification of the carboxylation product and determination of the acyl-CoA carboxylase components. J Biol Chem 280, 8862-8874.

Qin, Y. M., Haapalainen, A. M., Kilpelainen, S. H., Marttila, M. S., Koski, M. K., Glumoff, T., Novikov, D. K. \& Hiltunen, J. K. (2000). Human peroxisomal multifunctional enzyme type 2. Site-directed mutagenesis studies show the importance of two protic residues for 2enoyl-CoA hydratase 2 activity. J Biol Chem 275, 4965-4972.

Sambrook, J. \& Russell, D. W. (2001). Molecular Cloning: a Laboratory Manual, 3rd edn. Cold Spring Harbor, NY: Cold Spring Harbor Laboratory.

Sassetti, C. M., Boyd, D. H. \& Rubin, E. J. (2003). Genes required for mycobacterial growth defined by high density mutagenesis. $\mathrm{Mol}$ Microbiol 48, 77-84.

Schaeffer, M. L., Agnihotri, G., Volker, C., Kallender, H., Brennan, P. J. \& Lonsdale, J. T. (2001). Purification and biochemical characterization of the Mycobacterium tuberculosis $\beta$-ketoacyl-acyl carrier protein synthases KasA and KasB. J Biol Chem 276, 47029-47037.

Schroeder, E. K., de Souza, N., Santos, D. S., Blanchard, J. S. \& Basso, L. A. (2002). Drugs that inhibit mycolic acid biosynthesis in Mycobacterium tuberculosis. Curr Pharm Biotechnol 3, 197-225.

Smith, S., Witkowski, A. \& Joshi, A. K. (2003). Structural and functional organization of the animal fatty acid synthase. Prog Lipid Res 42, 289-317.

Snapper, S. B., Melton, R. E., Mustafa, S., Kieser, T. \& Jacobs, W. R., Jr (1990). Isolation and characterization of efficient plasmid transformation mutants of Mycobacterium smegmatis. Mol Microbiol 4, 1911-1919.

Stover, C. K., de la Cruz, V. F., Fuerst, T. R., Burlein, J. E., Benson, L. A., Bennett, L. T., Bansal, G. P., Young, J. F., Lee, M. H. \& other 
authors (1991). New use of BCG for recombinant vaccines. Nature 351, 456-460.

Takayama, K., Wang, C. \& Besra, G. S. (2005). Pathway to synthesis and processing of mycolic acids in Mycobacterium tuberculosis. Clin Microbiol Rev 18, 81-101.

Vilcheze, C., Morbidoni, H. R., Weisbrod, T. R., Iwamoto, H., Kuo, M., Sacchettini, J. C. \& Jacobs, W. R., Jr (2000). Inactivation of the inhAencoded fatty acid synthase II (FASII) enoyl-acyl carrier protein reductase induces accumulation of the FASI end products and cell lysis of Mycobacterium smegmatis. J Bacteriol 182, 4059-4067.

Wright, A., Bai, G., Barrera, L., Boulahbal, F., Martín-Casabona, N., Gilpin, C., Drobniewski, F., Havelková, M., Lepe, R. \& other authors (2006). Emergence of Mycobacterium tuberculosis with extensive resistance to second-line drugs-worldwide, 2000-2004. MMWR Morb Mortal Wkly Rep 55, 301-305.

Edited by: S. V. Gordon 